\title{
Vascular endothelial growth factor (VEGF)-C, VEGF-D, VEGFR-3 and D2-40 expressions in primary breast cancer: Association with lymph node metastasis
}

\author{
Aydan Eroğlu ${ }^{1, A-F}$, Cevriye Ersözz B, B, , Durdu Karasoy ${ }^{3, C}$, Serpil Sak ${ }^{2, A, C, E}$ \\ 1 Surgical Oncology Unit, Department of General Surgery, Ankara University Medical School, Ankara, Turkey \\ ${ }^{2}$ Department of Pathology, Ankara University Medical School, Ankara, Turkey \\ ${ }^{3}$ Department of Statistics, Faculty of Science, Hacettepe University, Ankara, Turkey \\ A - research concept and design; $B$ - collection and/or assembly of data; $C$ - data analysis and interpretation; \\ $D$ - writing the article; $E$ - critical revision of the article; $F$ - final approval of article
}

Address for correspondence

Aydan Eroğlu

E-mail: aydaneroglu@hotmail.com

Funding sources

Scientific Research Project of Ankara University (project no. 13B3330015)

Conflict of interest

none declared

Received on May 03, 2015

Revised on May 21, 2015

Accepted on July 10, 2015

\begin{abstract}
Background. Two members of the vascular endothelial growth factor (VEGF) family, VEGF-C and -D, are known as lymphangiogenic growth factors and play an important role in tumor lymphangiogenesis via activation of the VEGF receptor (VEGFR)-3, which is expressed in lymphatic endothelial cells. D2-40 is a specific antibody for lymphatic vessel density (LVD).

Objectives. In the present study, we have aimed to evaluate whether intra- and peri-tumoral D2-40-positive lymphatic vessels affect lymph node metastasis and to investigate the relationship between LVD and lymph node metastasis in breast cancer.

Material and methods. We have evaluated the relationships between lymph node metastasis and VEGF-C, VEGF-D, VEGFR-3 and D2-40 expressions in breast cancer cells using immunohistochemistry. VEGF-C, VEGF-D and VEGFR-3 expression were found in tumor cells in the majority of the cases $(83.75,97.5$ and $95 \%$, respectively).

Results. There was a significant positive relationship between VEGF-D expression and lymph node metastasis $(p<0.05)$ however no significant association was found in VEGF-C and VEGFR-3 expressions. It was found that patients with high-expression of VEGF-D have a high level of both peri- and intra-tumoral LVD compared to those with low expression of VEGF-D $(p<0.05)$.
\end{abstract}

Conclusions. Our results support that examination of VEGF-D expression in breast cancer cells may be beneficial in the identification of lymph node metastasis.

Key words: breast cancer, metastasis, VEGF, D2-40

DOI

10.17219/acem/58784

Copyright

Copyright by Author(s)

This is an article distributed under the terms of the

Creative Commons Attribution Non-Commercial License

(http://creativecommons.org/licenses/by-nc-nd/4.0/) 
Breast cancer is the most commonly diagnosed cancer in Turkish women as well as women worldwide. ${ }^{1}$ According to GLOBOCAN 2012, the most common cause of cancer mortality among women in Turkey is breast cancer. Although several molecular and clinicopathological factors have been investigated in breast cancer prognosis, lymph node status is still the most important prognostic factor. ${ }^{2}$ Lymph node-positive breast cancer is associated with poor prognosis as compared to node-negative tumors and also the number of involved lymph nodes is the most important and widely used independent prognostic factor in breast cancer. ${ }^{3}$

Breast cancer has a predilection to initially metastasize to the axillary lymph nodes, most commonly via the lymphatic system. ${ }^{4}$ Members of the vascular endothelial growth factor (VEGF) family, VEGF-C and VEGF-D are thought to be the lymphangiogenic factors that stimulate lymphangiogenesis via activation of VEGF receptor (VEGFR)-3 specifically expressed on lymphatic endothelial cells. ${ }^{5}$ Although the exact mechanisms and the role of lymphangiogenesis are unclear, VEGF-C and VEGF-D are believed to stimulate lymphangiogenesis. VEGFR-3 is expressed predominantly in the lymphatic endothelium and thus is considered to be a major regulator of lymphangiogenesis.

Staining of lymphatic endothelial cells by immunohistochemistry allows visualization of lymphatic vessels within and around tumors. D2-40/podoplanin is well known as one of the selective markers of lymphatic endothelial cells and therefore is used as a marker for identifying lymph vessels and detecting peri- and intra-tumoral lymphatic vessel density (LVD).

VEGF-C is considered to be a relatively specific lymphatic endothelial growth factor in normal tissues. Although VEGFR-3 is described as a predominantly lymphatic endothelial marker, Valtola et al. showed that VEGFR-3 is also very weakly expressed in the capillary endothelium of normal breast tissue. ${ }^{6}$ The authors demonstrated that both intraductal and invasive cancer cells contain VEGF-C protein. VEGF-C, VEGF-D and their binding to the cognate receptor VEGFR-3 in the regulation of lymphangiogenesis is accepted, but their value for lymph node metastasis in breast cancer is controversial. In a number of studies, elevated VEGF-C expression has been reported in 30-40\% of breast cancer. Although some studies have demonstrated that VEGF-C expression is significantly correlated with lymph node metastasis, some authors have found no correlation with lymphatic vessel invasion (LVI) or lymph node metastasis. ${ }^{7,8}$

The aim of this study was to investigate the possible relationships between VEGF-C, VEGF-D and VEGFR-3 expressions, LVD as determined by D2-40 and lymph node metastasis in primary invasive breast cancer. To the best of our knowledge, this is first study from Turkey which has evaluated the possible correlation between lymph node metastasis and the expression of VEGF-C, VEGF-D and VEGFR-3 and in breast cancer.

\section{Material and methods}

The study consisted of 80 female patients with invasive breast carcinoma (stage 1, stage 2 or stage 3 ), aged 31 80 years (mean $52 \pm 14$ years) who received primary surgical treatment by one surgical oncologist (A.E.) between 2008 and 2013 at the Department of Surgical Oncology, Ankara University Faculty of Medicine, and had sufficient paraffin-embedded tumor specimens. No patients had distant metastasis or received neoadjuvant chemotherapy or radiation therapy prior to surgery. The study was carried out with the approval of the Ethics Committee of Ankara University Faculty of Medicine (project no. 03-81-13) and a grant from the Scientific Research Project of Ankara University (project no. 13B3330015).

\section{Immunohistochemical staining}

For the 80 cases, the best paraffin block that showed the histological features of the tumor was selected; $4 \mu \mathrm{m} \mathrm{sec-}$ tions were cut and stained using the streptavidin-biotin peroxidase method on a "Ventana automatic immunostaining device" (BenchMark XT Staining Module, Ventana Medical Systems) for VEGFR-3 (ABCAM), VEGF-C (ABCAM), VEGF-D (ABCAM) and D2-40 (DAKO) at $1: 150,1: 20,1: 200$ and $1: 100$ dilutions, respectively (Table 1). The antibody dilutions and sources are shown in Table 1. Appropriate control tissues were included in each staining run.

Cytoplasmic staining in invasive tumor cells was considered as positive in the assessment of VEGFR-3, VEGF-C and VEGF-D staining. The staining results were evaluated using the immunoreactive score (IRS) proposed by Remmele and Stegner (9) with a slight modification as follows: staining intensity $(\mathrm{SI}) \times$ percentage of positive cells $(\mathrm{PP})$. SI was defined as $0=$ negative; 1 = weak; 2 = moderate; 3 = strong. PP was evaluated as $0=$ negative; $1=1-50 \%$ positive cells; $2=51-100 \%$ positive cells. An IRS of 0 to 2 was considered as low-expression. IRSs from 3 to 6 were accepted as high-expression. In the D2-40 evaluation, the number of D2-40 stained lymphatic vessels were counted on 10 intra-tumoral (located within the tumor) and 10 peri-tumoral (located in the adjacent non-tumoral peripheral tissue) high power fields (HPFs).

Table 1. Source and technical characteristics of antibodies

\begin{tabular}{|l|c|c|c|}
\hline Antibody & Clone & Dilution & Source \\
\hline VEGFR-3 & $\begin{array}{c}\text { ab51496 } \\
\text { mouse monoclonal }\end{array}$ & $1: 150$ & ABCAM \\
VEGF-C & $\begin{array}{c}\text { ab9546 } \\
\text { rabit polyclonal }\end{array}$ & $1: 20$ & ABCAM \\
VEGF-D & $\begin{array}{c}\text { ab155288 } \\
\text { rabit monoclonal }\end{array}$ & $1: 200$ & ABCAM \\
D2-40 & $\begin{array}{c}\text { M3619 } \\
\text { mouse monoclonal }\end{array}$ & DAKO \\
\hline
\end{tabular}




\section{Statistical analysis}

Between-group comparisons were evaluated using the Mann-Whitney U-test or ANOVA test. The Lease Significant Difference (LSD) test was used for the post-hoc tests to determine which specific groups differ significantly from one another. Mean differences in peri- and intra-tumoral LVD and IRS counts were compared with the use of the paired t-test. The $X^{2}$ test or Fisher's exact test were used to see if there was a relationship between 2 categorical variables. A p value $<0.05$ was considered to be statistically significant. The statistical analysis was done using IBM SPSS Statistics Premium 22 V (License manager name: spss.hacettepe.edu.tr).

\section{Results}

VEGFR-3 expression was observed in tumor cells in 76 of 80 (95\%) breast cancer patients. VEGF-C immunoreactivity was detectable in 67 (83.75\%) patients. VEGF-D expression in tumor cells was found in 78 (97.5\%) cases (Fig. 1).

Lymph node metastasis (N1 to N3) was positive in 51 (63.75\%) patients. No significant association was found between VEGF-C or VEGFR-3 expression and lymph node status. However, a significant positive relationship between VEGF-D expression and the presence of N2 or N3 lymph node metastasis was observed $(p<0.05)$. As seen in Table 2, patients with N2 and N3 lymph node metastasis had a significantly higher IRS for VEGF-D.

Intra-tumoral LVD ranged from 0 to 3, peri-tumoral LVD ranged from 1 to 12 (Fig. 2). To correlate LVD and VEGF-D expressions, the patients were divided into

Table 2. Relationship between lymph node status and mean VEGF-D IRS

\begin{tabular}{|l|c|c|}
\hline Lymph node status & VEGF-D (IRS) & p value* \\
\hline N0 & $2.69 \pm 0.26$ & 0.058 \\
N1 & $3.23 \pm 0.29$ & \\
N2 & $2.81 \pm 0.59$ & \\
N3 & $4.11 \pm 0.59$ & \\
\hline
\end{tabular}

* ANOVA test was used; $p=0.01$ for N0 vs N3, and $p=0.031$ for N2 vs N3, with LDS test

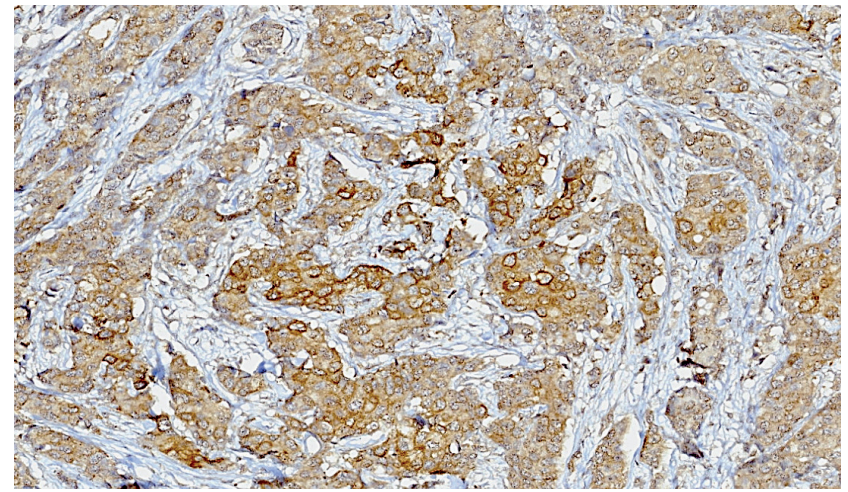

Fig. 1. Strong and diffuse VEGF-D staining in carcinoma cells, $\times 20$



Fig. 2. Lymphatic vessels in peri-tumoral area, D2-40, $\times 10$

2 groups with regard to VEGF-D expression: low-level of VEGF-D expression and high-level of VEGF-D expression. There were 10 patients with low-expression of VEGF-D and 70 patients with high expression of VEGF-D. As shown in Table 3, patients with high-expression of VEGF-D were observed to have high levels of both peri- and intra-tumoral LVD compared to those with a low level of VEGF-D expression. In addition, VEGFR-3 expression in tumor cells was found to be significantly correlated with peri-tumoral LVD ( $<$ 0.05), but not with intra-tumoral LVD (Table 3).

In addition to lymph node status, we evaluated the relationships between VEGF-C, VEGF-D and VEGFR-3 expressions and other clinicopathological variables. It was found that there were no significant correlation between VEGF-C, VEGF-D and VEGFR-3 expressions and the patient's age, tumor size, TNM stage or tumor grade (data not shown).

Table 3: Peri- and intra-tumoral lymphatic vessel density (LVD) according to the level of VEGF-D and VEGFR-3 expressions

\begin{tabular}{|c|c|c|c|c|}
\hline Level of VEGF expressions & Peritumoral LVD & $\mathrm{p}$-value* & Intratumoral LVD & $\mathrm{p}$-value* \\
\hline \multicolumn{5}{|l|}{ VEGF-D } \\
\hline low expression $(n=10)$ & $2.4 \pm 0.8$ & 0.002 & $0.8 \pm 0.4$ & 0.031 \\
\hline high expression $(n=70)$ & $5.5 \pm 3.1$ & & $1.29 \pm 0.7$ & \\
\hline \multicolumn{5}{|l|}{ VEGFR-3 } \\
\hline low expression ( $n=12)$ & $3.1 \pm 2$ & 0.014 & $1.2 \pm 0.8$ & 0.650 \\
\hline high expression ( $n=68$ ) & $5.5 \pm 3.1$ & & $1.3 \pm 0.6$ & \\
\hline
\end{tabular}

* Mann-Whitney U test was used. 


\section{Discussion}

It is known that breast cancer may spread via the lymphatic vessels into the axillary lymph nodes. ${ }^{4}$ Lymphatic vessel invasion (LVI) has also been shown to significantly correlate with lymph node metastasis as well as sentinel and non-sentinel lymph node metastasis. ${ }^{10}$ VEGF-C and VEGF-D are known as lymphangiogenic factors; both growth factors mediate their biological activity mainly by VEGFR-3. ${ }^{11}$

Although the relationships of VEGF-C and -D with lymph node metastasis are still a controversial issue, they are generally accepted to be significantly associated with the extent of lymph node metastasis in breast cancer. ${ }^{12}$ Bando et al. evaluated intra-tumoral levels of VEGF-C and VEGFR-3 by using enzyme-linked immunosorbent assay in human breast cancer tissues and found that there was no significant prognostic value of VEGFR-3. ${ }^{11}$ Recently, some authors have demonstrated that different molecular subtypes of breast cancer correlate with the expression of VEGF-C and/or VEGF-D. ${ }^{13,14}$ Raica et al. investigated the differential of expressions VEGF-C, VEGFR-3 and D2-40 by immunohistochemistry to evaluate lymphangiogenesis and the lymphatic microvessel density in breast cancer patients. ${ }^{13}$ They found that VEGF-C expression significantly correlated with lymph node metastasis, but not tumor grade. The authors showed the highest level of VEGF-C expression in HER2 and luminal types and the lowest expression in basal-like carcinoma. In contrast, Liu et al. showed that triple-negative breast cancer correlates with a high expression of VEGF-C and -D. ${ }^{14}$

Although some researchers have suggested that VEGF-C, but not VEGF-D, has an important role in lymphangiogenesis, others believe that VEGF-D is a predictor for lymphatic metastasis, and an independent prognostic factor. ${ }^{15-19}$ The first group of researchers have suggested that VEGF-C promotes lymphangiogenesis and that tumor lymphangiogenesis in turn promotes lymphatic metastasis. ${ }^{15,16,20}$ In the present study, we showed that VEGF-C, VEGF-D, and VEGFR-3 were expressed in a substantial percentage of breast carcinomas. VEGF-D expression was associated with lymph node metastasis, however there were no significant correlations between the expressions of VEGF-C and VEGFR-3 and lymph node status. In a study by Nakamura et al., LVD was evaluated by podoplanin immunostaining and the relationships between LVD and lymph node status as well as VEGF-C immunoreactivity and some clinicopathological parameters were analyzed. ${ }^{20}$ The authors found that increased LVD was correlated with lymph node metastasis and VEGF-C expression. In their series, peri-tumoral lymph vessels were slightly higher in number when compared to intra-tumoral lymph vessels. Similarly, we have also observed that peri-tumoral lymph vessels were significantly higher than intra-tumoral lymphatic vessels in our study.
Contrary to the findings of the previous studies demonstrating that high expression of VEGF-C in human breast cancer is significantly correlated with lymph node metastasis and unfavorable prognosis, we have not observed any correlation of VEGF-C expression in tumor cells with lymph node status. In some studies, peri-tumoral LVD was found to be an independent risk factor for axillary lymph node metastasis. ${ }^{10,21}$ In a recent study, VEGF-C and -D expressions were significantly associated with peritumoral LVD, as determined by D2-40 staining, but not intra-tumoral LVD in breast cancer. ${ }^{22}$ The authors have found that peri-tumoral LVD was associated with lymph node metastasis, LVI and advanced tumor stage and thus they suggested that tumor derived VEGF-C and -D induce peri-tumoral lymphangiogenesis, which may be related to lymphatic invasion and metastatic spread. Mohammed et al. showed that high expressions of VEGF-A and -C, but not of VEGF-D, were associated with a higher LVD and lymph node metastasis. ${ }^{16}$ In another study by $\mathrm{Gu}$ et al., LVD was found to be significantly associated with lymphatic metastasis and advanced tumor stage. ${ }^{17}$ In their series, increased LVD was significantly correlated with high expressions of VEGF-C and -D. In their study, there was a close correlation between VEGF-D expression and lymphatic metastasis. In our study, VEGF-D expression was found to be significantly associated with both peritumoral and intra-tumoral LVD. There was also a significant correlation between VEGF-D expression and lymph node metastasis. We have observed that peri-tumoral, but not intra-tumoral, LVD was closely related to the high expression of VEGFR-3 in breast cancer cells.

In summary, our findings suggest that high level VEGF-D expression by breast cancer cells may induce lymphangiogenesis in the peri-tumoral and intra-tumoral region and thus contribute to a high peri- and intra-tumoral LVD, leading to lymphatic vessel invasion and metastatic spread to lymph nodes. According to the previous published studies and our present series, we can suggest that inhibition of VEGF-D expression to control peri- and intra-tumoral lymphangiogenesis may contribute to the development of novel therapeutic strategies for breast cancer treatment. ${ }^{23}$

\section{References}

1. Globocan 2012: Estimated cancer incidence, mortality and provalence. http://globocan.iarc.fr/Pages/fact_sheets_population.aspx. Accessed 2015.

2. Untch M, Gerber B, Harbeck N, et al. $13^{\text {th }}$ St. Gallen International Breast Cancer Conference 2013: Primary therapy of early breast cancer evidence, controversies, consensus-opinion of a German team of experts. (Zurich 2013). Breast Care (Basel) 2013;8:221-229.

3. Yang C, Liu F, Liu S, Li W, Zhai L, Ren M. Lymph node ratio. A new feature for defining risk category of node-positive breast cancer patients. Int J Surg Pathol. 2012;206:546-554.

4. Pepper MS. Lymphangiogenesis and tumor metastasis: More questions than answers. Lymphology 2000;33:144-147.

5. Auwera IV, Cao Y, Tille JC, et al. First international consensus on the methodology of lymphangiogenesis quantification in solid human tumours. Br J Cancer. 2006;95:1611-1625. 
6. Valtola R, Salven P, Heikkila P, et al. VEGFR-3 and its ligand VEGF-C are associated with angiogenesis in breast cancer. Am J Pathol. 1999;154:1381-1390.

7. Koyama Y, Kaneko K, Akazawa C, et al. Vascular endothelial growth factor-C and vascular endothelial growth factor-D messenger RNA expression in breast cancer: Association with lymph node metastasis. Clin Breast Cancer. 2003;4:354-360.

8. Hoar FJ, Chaudhri S, Wadley MS, Stonelakc PS. Co-expression of vascular endothelial growth factor C (VEGF-C) and c-erbB2 in human breast carcinoma. Eur J Cancer. 2003;39:1698-1703.

9. Remmele W, Stegner HE. Recommendation for uniform definition of an immunoreactive score (IRS) for immunohistochemical estrogen receptor detection (ER-ICA) in breast cancer tissue. Pathology. 1987:8:138-140.

10. Ran S, Volk L, Hall K, Flister MJ. Lymphangiogenesis and lymphatic metastasis in breast cancer. Pathophysiology. 2010;17:229-251.

11. Bando $\mathrm{H}$, Weich HA, Horiguchi $\mathrm{S}$, et al. The association between vascular endothelial growth factor- $C$, its corresponding receptor, VEGFR-3, and prognosis in primary breast cancer: A study with 193 cases. Oncology Reports. 2006;15:653-659.

12. Skobe M, Hawighorst T, Jackson DG, et al. Induction of tumor lymphangiogenesis by VEGF-C promotes breast cancer metastasis. Nat Med. 2001;7:192-198.

13. Raica M, Cimpean AM, Ceausu R, Ribatti D. Lymphatic microvessel density, VEGF-C, and VEGFR-3 expression in different molecular types of breast cancer. Anti Cancer Res. 2011;31:1757-1764.

14. Liu HT, Ma R, Yang QF, Du G, Zhang CJ. Lymphangiogenic characteristics of triple-negative in node-negative breast cancer. Int J Surg Pathol. 2009;17:426-431.
15. Swartz MA, Skobe M. Lymphatic function, lymphangiogenesis, and cancer metastasis. Microsc Res Tech. 2001;55:92-99.

16. Mohammed RAA, Green A, El-Shikh S, et al. Prognostic significance of vascular endothelial growth factors -A, $-C$, and -D in breast cancer and their relationship with angio- and lymphangiogenesis. Br J Cancer. 2007;96:1092-1100.

17. Gu Y, Qi X, Guo S. Lymphangiogenesis induced by VEGF-C and VEGF-D promotes metastasis and a poor outcome in breast carcinoma: a retrospective study of 61 cases. Clin Exp Metastasis. 2008; 25:717-725.

18. Yasuoka H, Nakamura Y, Zuo H, et al. VEGF-D expression and lymph vessels play an important role for lymph node metastasis in papillary thyroid carcinoma. Med Pathol. 2005;18:1127-1133.

19. Yonemura $Y$, Endo $Y$, Tabata $K$, et al. Role of VEGF-C and VEGF-D in lymphangiogenesis in gastric cancer. Int J Clin Oncol. 2005;10:318-327.

20. Nakamura $Y$, Yasuoka $H$, Tsujimoto $M$, et al. Lymph vessel density correlates with nodal status, VEGF-C expression, and prognosis in breast cancer. Breast Cancer Res Treat. 2005;91:125-132.

21. Britto AV, Schenka AA, Moraes-Schenka NG, et al. Immunostaining with D2-40 improves evaluation of lymphovascular invasion, but may not predict sentinel lymph node status in early breast cancer. BMC Cancer. 2009;9:109.

22. Zhao YC, Ni XJ, Li Y, et al. Peritumoral lymphangiogenesis induced by vascular endothelial growth factor-C and D promotes lymph node metastasis in breast cancer patients. World J Surg Oncol. 2012; 10:165.

23. Dvorak HF, Weaver VM, Tlsty TD, Bergers G. Tumor microenvironment and progression. J Surg Oncol. 2011;103:468-474. 